\title{
Congenital Cytomegalovirus Infection: Transmission, Diagnosis and Treatment
}

\author{
Muhammad Romail Manan ${ }^{1}$ | Hamna Manan ${ }^{2}$
}

${ }^{1}$ Services Institute of Medical Sciences, Lahore, Punjab, Pakistan

2 Jinnah Hospital, Lahore, Punjab, Pakistan

\section{Correspondence}

Muhammad Romail Manan

Email: romailmanan1@gmail.com

\section{Publication Date}

October 1, 2021

\section{MJM 2022 (20) 10}

https://doi.org/10.26443/mjm.v20i1.876

\section{McGill Journal of Medicine}

www.mjmmed.com

\section{(1) (2)}

This work is licensed under a Creative Commons BY-NC-SA 4.0 International License.

\section{ABSTRACT}

Introduction: Cytomegalovirus (CMV) is a linear, dsDNA virus that is regarded as the prototype of the Betaherpesvirinae subfamily of viruses. It has an established endemic status in certain locations around the globe and is also reported to be the most prevalently occurring congenital infection in humans. Furthermore, Cytomegalovirus is notorious for being a persistent lifelong pathogen that poses a threat of reactivation as well.

Discussion: Congenital cytomegalovirus infection causes numerous ophthalmologic, and neurologic sequelae, and is also known for being the principal reason behind sensorineural hearing loss of non-genetic etiology in neonates. These symptoms, if present, may give rise to a premonition of congenital Cytomegalovirus disease, and so, a diagnosis can be established through serology, radiology, and PCR of salivary, urinary, or dried blood spot samples. Timely administration of ganciclovir or valganciclovir has proven to be effective in managing symptomatic cases of congenital CMV.

Conclusion: A well-timed delivery of pharmacological and nonpharmacological interventions is necessary to achieve healthy developmental outcomes for the neonate. Moreover, there is still a need to study the role of antiviral therapy in silent cases since asymptomatic patients are at risk of developing long-term clinical sequelae as well.

Relevance: An estimated $60-90 \%$ of women of child-bearing age get infected with Cytomegalovirus, and Congenital CMV disease is reported in $0.2-2.4 \%$ of all live births. Therefore, in order to develop effective screening and management protocols, it is vital to educate healthcare professionals regarding the various aspects of this congenital infection.

\section{KEYWORDS}

Congenital, Cytomegalovirus, Infection, Hearing loss 


\section{1 | INTRODUCTION}

Cytomegalovirus, also known as the Human betaherpesvirus 5 , is a pathogen that is indexed under a family of viruses known as the 'Herpesviridae', and it is also regarded as the prototype of the Betaherpesvirinae subfamily. Its linear, double-stranded(ds) DNA genome is considered to be one of the largest amongst the other human viruses; and, possessing a length of approximately $235 \mathrm{kbp}$, it is also recognized as the largest genome amongst the human herpesviruses. (1) CMV is unanimously accredited as the most prevalently occurring congenital infection in humans. It is reported to infect $60-90 \%$ of women of child-bearing age in various parts of the world. (2) The global circumstances surrounding CMV infections are recognized as being of an endemic nature owing to the understanding that CMV infections fester in most of the human populations. (2) Moreover, the seroprevalence of CMV is generally subject to the socioeconomic status of the affected area. Therefore, incidences reaching a maximum of $100 \%$ may be observed in underdeveloped locations. (3) CMV mainly causes an asymptomatic primary infection in immunocompetent people but may manifest as a severe focal disease having various clinical symptoms in immunosuppressed individuals. As observed with other herpesviruses, the phenomenon of latency and reactivation is also prominently discerned in cases of CMV infection. Therefore, persistent lifelong infection with the risk of potential reactivation on encountering a breach in the immune defense mechanisms is a characteristic clinical challenge associated with these viruses. (3)

This review aims to explore the various aspects of congenital CMV infection including transmission, clinical manifestations, diagnosis, and treatment. Moreover, while discussing these facets, a primary focus will remain on the recent diagnostic and therapeutic developments recorded in the literature.

\section{IRANSMISSION}

Primary CMV infection in a healthy individual is generally silent but the shedding of the virus may per- sist through urine, saliva, and blood. Therefore, the spread of the virus is maintained through sexual and nonsexual contact. (4) Intimate degrees of contact with an infected individual capable of potentially expelling the virus through their bodily secretions appears to be the primary mechanism of horizontal CMV transmission. (5) Transplantation of an organ-harvesting latent CMV and transmission of CMV through blood transfusions in immunosuppressed individuals can result in a lifethreatening disease as well. (5) However, it appears that monocytes serve as prime vectors for latent CMV and, hence, the transfusion of leukocyte-reduced blood can lower the incidence of transfusion-transmitted CMV disease in immunocompromised individuals. (6)

Moreover, vertical transmission plays a pivotal role in maintaining a sustained incidence of human CMV infections. Mothers who are infected during or even before pregnancy can transmit the infection to their child. Three routes of vertical CMV transmission have been primarily described, (7)

1. Transplacental route during the intrauterine period

2. Through breast milk

3. Intrapartum route

It should be noted that only transplacental route of CMV transmission results in a congenital infection while intrapartum and postpartum transmission does not cause congenital CMV disease.

The frequency of transplacental transmission of CMV varies with the gestation period. The rate of transmission progresses from a lower incidence of $20 \%$ to a higher incidence of $75 \%$ if the primary infection is contracted during the first and third trimester respectively. (7) Furthermore, congenital CMV disease as a consequence of first-trimester maternal infection, is more likely to manifests as a symptomatic case at birth with the possibility of resulting in long term disabilities. $(8,9)$ Additionally, it should be noted that previously acquired maternal immunity against CMV is not always efficacious in preventing congenital CMV in the neonate. Evidence exists to suggest that maternal reinfection with a CMV strain possessing a slightly different epitope 
could potentially explain why children of women with past immunity would develop the congenital CMV infection. (10) Hematogenous spread of infected leukocytes across the placental barrier, and local infection of the placenta and amniotic fluid are mechanisms that describe the transplacental spread of CMV. (11)

Additionally, reactivation of CMV is observed in $96 \%$ of the seropositive lactating women, and the infectivity of breast milk can be established 3 days post-delivery. (12) There have been reports in the literature to suggest that postnatally acquired CMV infections can yield severe consequences in some premature infants. (1315) Therefore, efforts have been made to develop techniques that can aid the removal of CMV from milk while also conserving its beneficial elements. Processes to treat the milk at high temperatures $\left(72^{\circ} \mathrm{C}\right)$ for a short duration of time ( 5 seconds) have proven to be somewhat effective in achieving the desired degree of preservation. (16) Alternatively, milk donated by seronegative lactating women can also be utilized to avoid the potential risks of losing the health-giving properties of colostrum when subjected to such treatments. (17)

\section{3 | CLINICAL MANIFESTATIONS OF CONGENITAL CYTOMEGALO- VIRUS INFECTION}

An estimated $0.2-2.4 \%$ of all live births suffer from a congenital infection of cytomegalovirus. (18) Additionally, congenital CMV infection is reported to be the most common non-genetic reason behind sensorineural auditory impairment and delay in neurological development in children. (18) About $90 \%$ of the newborns infected with CMV have a clinically silent infection and show no perceivable symptoms or signs. (18) However, up to $56 \%$ of these apparently asymptomatic patients may have laboratory, ophthalmologic, or neuroimaging findings that would recategorize them as symptomatic cases when evaluated. (18) Therefore, a well-oriented, thorough and targeted screening approach is necessary so as to not miss any instances of infection in the apparently healthy neonates. (18)
Infants with asymptomatic CMV infection may not have the typical physical or neurodevelopmental abnormalities, (19) but the literature reports that up to $23 \%$ of these asymptomatic children may experience sensorineural hearing loss, (20) which can hinder their normal linguistic and cognitive development. (21)

Hearing impairment, hepatomegaly, splenomegaly, petechial rash, thrombocytopenic purpura, jaundice, growth retardation, chorioretinitis, and cataracts are some of the presenting signs observed in a neonate suffering from congenital CMV disease. (22) Moreover, a placenta that is 3-folds greater in size than average has also been observed if the neonate has acquired the congenital CMV infection. (23)

\section{1 | Laboratory Findings}

An increase in serum transaminases, decreased platelet count, and increased serum concentration of conjugated bilirubin are the most frequently occurring laboratory manifestations of congenital CMV infection. (24) Platelet counts below 50,000/ $\mu \mathrm{L}$ have been observed in $1 / 3$ rd of the symptomatic patients. (25) CMV-related thrombocytopenia may be observed due to the destruction of platelets via autoimmune mechanisms, bone marrow dysfunction, or the consumption of platelets as a result of disseminated intravascular coagulation. Furthermore, bone marrow dysfunction or hemolytic destruction of blood cells may also result in mild anemia. Polychromasia, increased reticulocyte count, and RBCs possessing nuclei, are some indications suggestive of a hemolytic etiology. Moreover, bone marrow examination may reveal a decreased erythrocyte precursor count if the virus induces bone marrow inhibition as well as, an elevated erythroid to myeloid ratio is detected in circumstances of hemolysis. (25) It has also been reported that $50 \%$ of the symptomatic CMV patients may have elevated cerebrospinal fluid (CSF) proteins with lymphocytic pleocytosis and CMV may even be detectable in the CSF of these patients. $(25,26)$ 


\section{2 | Sensorineural Hearing Loss}

The sectional temporal bone analysis during the autopsy examinations of children suffering from congenital CMV infection has revealed the presence of viral inclusion bodies throughout the inner ear. (27) Diffusely present viral inclusion bodies have been discerned in the basilar membrane, cochleovestibular ganglion, Reissner's membrane, and spiral ligament. Destruction of the inner hair cells along with the outer hair cells has also been detected and so, a few theories have tried to explain the reasons behind the loss of these cell populations responsible for the transduction of auditory signals. Inflammatory response of inner ear to $\mathrm{CMV}$, direct infection of the hair cells, or CMV mediated destruction of stria vascularis are possible mechanisms operating behind the loss of hair cells and ultimately, behind the sensorineural auditory impairment associated with congenital cytomegalovirus infection. (28)

CMV-associated hearing impairment can be a finding detected at birth or it may even be identified later in life owing to a delay in its onset. Up to $50 \%$ of sensorineural hearing loss ( $\mathrm{SNHL}$ ) due to congenital cytomegalovirus infection is delayed-onset loss of hearing, and almost half of these patients experience progressively deteriorating auditory function. (29) Late onset hearing loss due to congenital CMV infection may occur a few years after birth, and a delay of 11 months in the onset of SNHL is generally observed in asymptomatic patients compared to the symptomatic cases. (29) Therefore, children with congenital CMV infection should have a periodic evaluation of their hearing until the age of about 6 years. (29). It is documented that approximately $5 \%$ of the asymptomatic patients develop at least unilateral SNHL within 12 months of being born. (30) CMV-related congenital sensorineural hearing loss is generally not observed if the maternal infection occurred during the 3rd trimester of pregnancy. While, $80 \%$ of the children born to mothers primarily infected in the first trimester had sensorineural auditory impairment at birth. (31) It is, however, interesting to note that fluctuation in the sensorineural hearing loss is a factor independent of the gestation period. (31)
Vigilant identification of auditory impairment early in life, and initiation of nonpharmacological interventions within a few months can substantially aid linguistic, social, behavioral and emotional child development. (32) Ideally, the recognition of hearing impairment and appropriate intervention should begin before 6 months to achieve desirable degrees of child development. (33) Healthier child developmental outcomes are also vital in relieving parental stress and maintaining a nourishing parent-child interaction. (32)

\section{3 | Ophthalmologic Manifestations}

Approximately $5-30 \%$ of patients with congenital CMV disease have ophthalmologic manifestations. (34) Results of a long-term prospective study have highlighted that $78 \%$ of the symptomatic congenital CMV patients had normal vision compared to $98.8 \%$ of the asymptomatic cases. (34) $1.2 \%$ of the patients in the asymptomatic group had moderate vision loss caused by macular scarring while $17 \%$ of the symptomatic patients had severe vision loss owing to cortical blindness or optic atrophy. (34) Ocular lesions of congenital CMV disease can be categorized as per the anatomical structures of the eye. It has been estimated that the lesions of anterior segment of the eye are not usually a consequence of congenital CMV infection. (34) This finding hints towards the minimal capacity of the cytomegalovirus to affect non-neuronal cellular lineages. (34) Moreover, the posterior segment of the eye and the cortical visual pathway are accounted as the potential sites for $\mathrm{CMV}$ action in children. (34) Chorioretinitis, optic atrophy, strabismus, and visual cortex lesions are the abnormalities that are most frequently detected, (34) and optic atrophy as well as cortical visual impairments have been reported to be the most frequent causes of bilateral visual defects in such patients. (35) Additionally, it should be noted that active cases of CMV-related retinitis can present with or without hemorrhagic manifestations. (35) Since ocular developmental abnormalities can also be seen as a consequence of congenital CMV infection, a targeted investigation of all severe developmental abnormalities of the eye might appear necessary but is generally not 
required unless other clinical signs of congenital CMV disease are also present. (35)

\section{4 | Neurologic Manifestations}

It is generally perceived that symptomatic cases of congenital CMV infection are associated with poor neurological outcomes in the patient. However, a study has reported that $59 \%$ of the children suffering from symptomatic congenital CMV infection had a normal Intelligence Quotient (IQ). (36) However, microcephaly has been reported to be a firm indicator of poor cognitive developmental outcomes in a child suffering from congenital CMV infection. (36) Similarly, lissencephaly and pachygyria are also linked to more severe neurological disabilities as compared to polymicrogyria. (37) Some of the other neurological presentations may include lethargy, seizures, hypotonia and poor oral feeding. (24) It has been estimated that neurological impairments are detectable in $6.5 \%$ of the asymptomatic patients and a total of approximately 8000 children in the US suffer from CMV-related neurological impairments each year. (37)

A neuropathogenic model hypothesizing the development of neurologic manifestations of CMV has been proposed by studying the spread of CMV particles in the brain of mice. CMV initially causes meningitis and choroid plexitis by diffusing out of the meningeal and choroidal vessels. (38) This spread, in turn, leads to a blood brain barrier disruption, and therefore, cytomegalovirus particles further spread to infect the ventricular and subventricular zones where the virus induces a neural cell loss by blocking the proliferation and differentiation of neural stem progenitor cells. (38)

\section{5 | Findings on Neuroimaging}

Bedside ultrasound, MRI, and CT scan are the imaging technologies employed in order to obtain an elaborate evaluation of the newborn suspected of having congenital cytomegalovirus infection. Ventricular size determination, as well as the visualization of periventricular calcifications can be achieved with the aid of ultrasound technology. Periventricular calcifications along with neuronal migration abnormalities are among the classical findings discerned in a patient of congenital CMV infection, and these abnormalities also serve to differentiate congenital CMV infection from other congenital diseases. Ultrasound can be appropriately utilized as the first imaging procedure in symptomatic children and it serves as a reasonable technique for predicting the outcomes in such patients as well. (39) Furthermore, head CT scan provides a more detailed image that is beneficial in effective localization and depiction of the cranial manifestations such as calcifications. (37) Cerebellar hypoplasia, polymicrogyria, lissencephaly, schizencephaly, ventriculomegaly, and cortical dysplasia are some of the neurodevelopmental manifestations that can be visualized effectively using MRI technology. (37) However, ultrasound may be more effective in the visualization of periventricular calcifications (even prenatally) as compared to an MRI. (37)

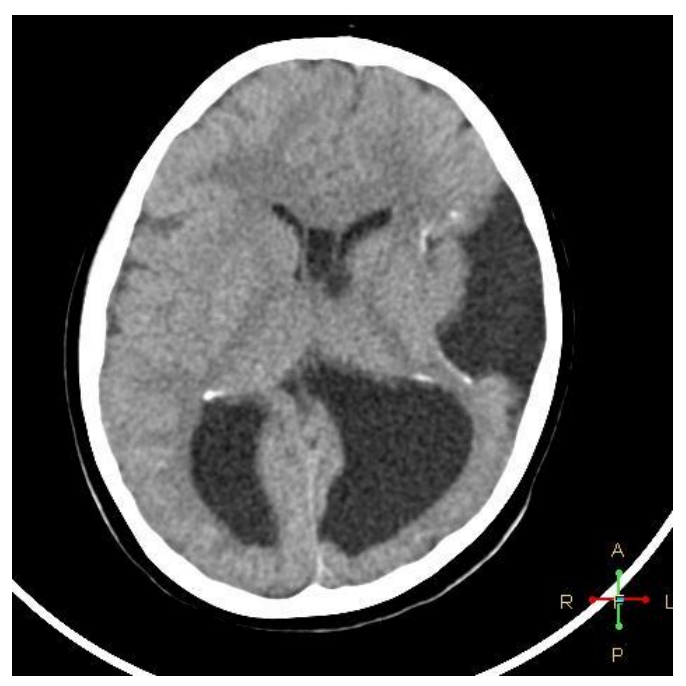

FIGURE 1 10-year-old male presented with delayed mental and motor milestones. Axial non-contrast computed tomography scan shows hydrocephalus with periventricular calcification, pachygyria, and cavum septum pellucidum. Case courtesy of Dr Ahmed Abdrabou, Radiopaedia.org, rID: 24282. 


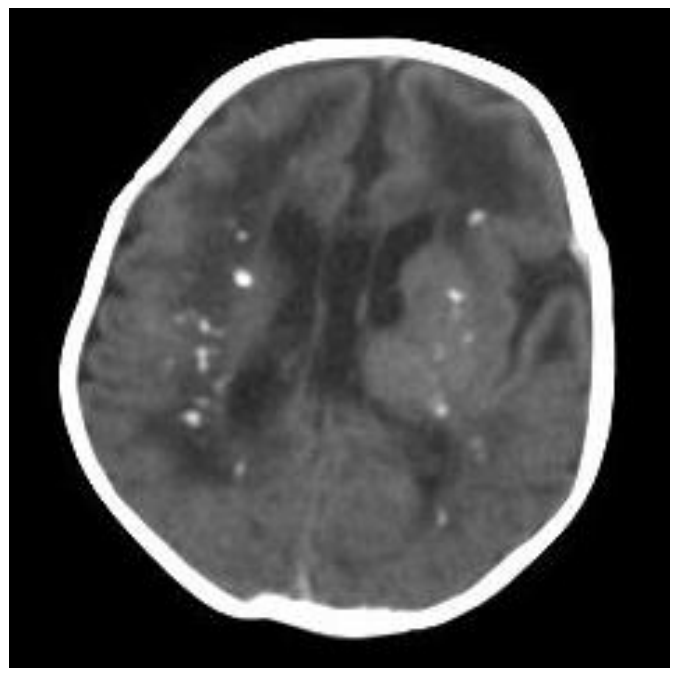

FIGURE 2 Computed tomography scan of a 2-month-old male showing scattered periventricular and basal ganglia calcification. These findings may be indicative of congenital cytomegalovirus infection. Case courtesy of Dr Aneesh KM, Radiopaedia.org, rID: 17105.

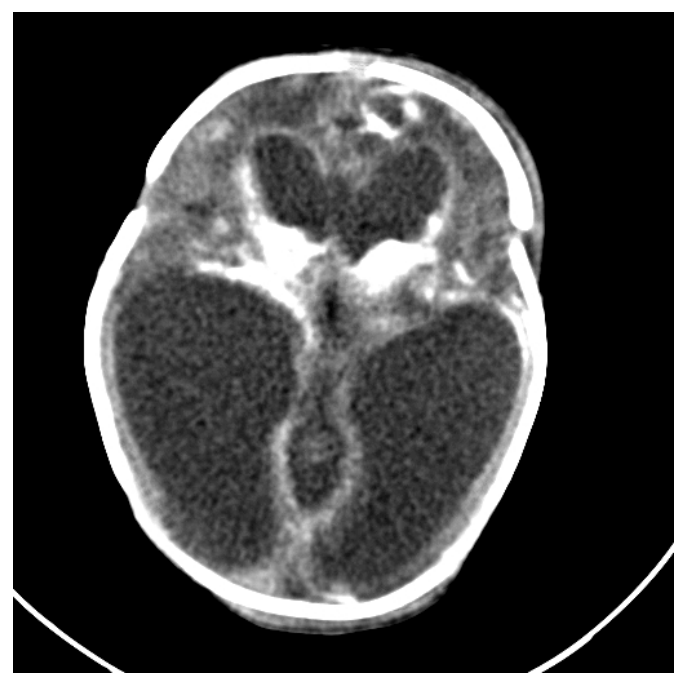

FIGURE 3 1-day-old male has scalp swelling and a weak cry. Extensive periventricular, basal ganglionic and parenchymal calcification with hydrocephalic changes and parenchymal atrophy can be seen on the computed tomography scan.

Case courtesy of Dr Ahmed Abdrabou, Radiopaedia.org, rID: 29095

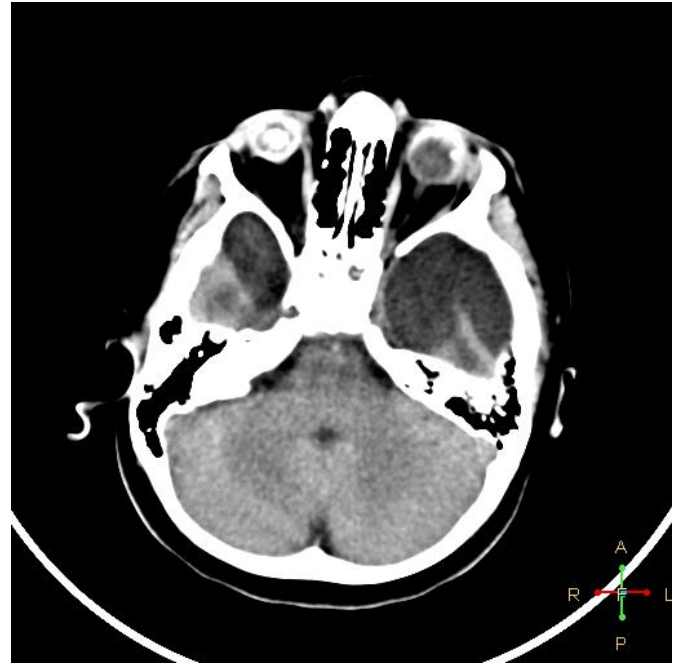

FIGURE 4 10-year-old male presented with delayed mental and motor milestones. Axial non-contrast computed tomography shows right microphthalmia and bilateral chorioretinal calcification. Case courtesy of Dr Ahmed Abdrabou, Radiopaedia.org, rID: 24282.

\section{4 | DIAGNOSIS}

Congenital CMV is a clinically significant infection that prevails substantially and is also documented under notorious terms for its long-term sequelae that can potentially hinder the normal development of children. Despite the incidence associated with congenital CMV infection, cases often go unidentified due to asymptomatic manifestation or nonspecific symptoms at birth. As discussed earlier, late-onset sequelae such as SNHL can be observed in both symptomatic and asymptomatic patients, highlighting the need for a post-natal screening protocol for congenital cytomegalovirus infection. The development of a screening protocol mostly relies on the clinical manifestation of a disease, but due to its potential asymptomatic presentation, an evident risk of missing the majority of the congenital CMV cases is highlighted to be problematic. Therefore, emphasis has been made recently on the need for a universal screening protocol. Currently, a lack of recommendations and effective implementation of appropriate 
universal neonatal screening protocols are observed in many countries, (40) since most asymptomatic cases of congenital CMV do not lead to clinical sequelae, and a diagnosis of congenital CMV infection could unreasonably stress the parents. Moreover, a lack of treatment options available to prevent sequelae in asymptomatic group makes the implementation of a universal screening protocol even more difficult. However, it is worth mentioning that Ontario, Canada has implemented universal screening for CMV as part of their Infant Hearing Program, and a study has also indicated the feasibility of upscaling the Australian pilot CMV screening program into a universal newborn hearing screening (UNHS) program. (41)

Universal CMV screening of neonates has yet to find implementation in most countries but efforts to study and implement targeted screening of neonates who fail the audiological screening examination have proven to be beneficial and cost effective to some extent in the United States, United Kingdom, Belgium, and Australia, (42-45) however such targeted screening can miss the majority of the asymptomatic cases.

Maternal CMV testing is usually indicated after uncovering suspicious fetal ultrasound findings such as fetal ventriculomegaly, growth restriction, cerebral calcifications, and echogenic fetal bowels. (46) Therefore, universal maternal screening for CMV is not recommended unless suspicious pre-natal ultrasound findings are reported. Similarly, symptoms of primary CMV infection in the pregnant women can also serve as an indicator for maternal CMV testing.

Maternal testing for CMV immunoglobulin M (IgM) is the most prevalently deployed investigation to detect primary maternal infection, but IgM may even be positive in cases of reinfection or reactivation. CMV IgM peaks in the initial 1-3 months and may persist for up to 12 months. Moreover, false positive IgM results are not infrequent in the presence of some other autoimmune pathology or viral infection. Due to the difficulties associated with interpreting IgM results, serum immunoglobulin $\mathrm{G}$ (IgG) avidity can be a beneficial investigation to differentiate between primary maternal infection and reinfection or reactivation. Avidity testing of antibodies detects the strength of binding between the polyvalent antibody and the antigen. Serum IgG detected up to 18 weeks after a primary maternal infection has a low to moderate avidity, while high avidity IgG may persist for years. (46) Therefore, high avidity is only detectable in past CMV infections. When jointly interpreted, low avidity of maternal IgG, and detectable IgM in the mother's blood can be firm indicators of a positive primary maternal infection. (46) Hence, it is not recommended to base the diagnosis of primary CMV infection solely on a detectable lgM, since avidity testing of lgG appears to be necessary to diagnose a primary maternal infection acquired within the past 3 months. (46)

Saliva and urine are the ideal samples to detect the presence of CMV in a neonate, however, oral swabs are preferred over urine samples merely due to convenience in their collection. (47) CMV polymerase chain reaction (PCR) is now increasingly preferred over CMV cultures for screening and diagnostic purposes and PCR testing of saliva has also been validated as an appropriate screening method. (47) Timely collection of these samples is crucial to detect congenitally acquired CMV, since positive CMV indications in samples collected 3 weeks post-delivery may represent a postnatally acquired infection which is usually not associated with significant clinical sequelae. (48) Similarly, false positive results can also be seen if the salivary sample is taken after the child is recently breastfed but it is important to note that the rate of false positive results with salivary samples is still reported to be considerably low. Additionally, stored and dried blood spots obtained at birth can also be useful to retrospectively diagnose a congenital CMV infection, but the sensitivity of this technique is documented to be significantly lower than the one for PCR testing of salivary samples. (48) Moreover, only $80-90 \%$ of the newborns with congenital CMV disease have the virus particles in their blood soon after birth, therefore a negative dried blood spots test cannot completely rule out congenital Cytomegalovirus disease. (48) Undetectable cytomegalovirus-specific IgG effectively discounts the probability of a congenital infection. (47) Serum IgM level of an infant has a low sensitivity in diagnosing congenital CMV infection, but an elevated level of serum 
IgM can serve as an indicator for symptomatic disease, thus encouraging the healthcare providers to aptly initiate further investigations for such patients. (49) However, it should be noted that serum IgM testing has no utilization in ruling out symptomatic disease. (49)

Prenatal identification of CMV is equally crucial to plan potentially necessary therapeutic measures and to also provide timely informational care regarding the potential risks of congenital CMV infection to the family. Amniocentesis for the detection of CMV DNA is the most effective tool in diagnosing congenital CMV infection prenatally. (50) This diagnostic amniocentesis should be performed ideally after 21 weeks of gestation and 6 weeks after the primary maternal infection. (46) Risk of a false negative result has been reported if the amniocentesis is performed prior to the 21st gestational week. (46)

\section{5 | TREATMENT}

Antiviral therapy for congenital CMV infection is only indicated in symptomatic cases and treatment of asymptomatic cases at birth has not shown to reduce SNHL later in life. Numerous developments have been recently recorded to establish the utilization of ganciclovir and oral valganciclovir for the management of symptomatic congenital cytomegalovirus cases. According to the results of a randomized control trial, early administration (within 1 month) of ganciclovir at a dose of $6 \mathrm{mg} / \mathrm{kg}$ administered twice daily for 6 weeks, demonstrated hearing improvement at 6 months and also successfully prevented further deterioration in hearing. (51) Similarly, improvements in neurodevelopment at 6 months and 12 months have also been suggested with the use of ganciclovir. (52) However, a momentous proportion of ganciclovir-associated hematotoxic adverse effects have been documented in these patients. $63 \%$ of the trial subjects developed severe neutropenia during treatment, (51) and even further, toxicity of the gonads and carcinogenic properties of ganciclovir have also been studied in animal subjects. (51)

Oral valganciclovir has established a comparable ef- ficacy to ganciclovir with fewer adverse effects and convenient administration, but valganciclovir has not proven to be beneficial in improving short term hearing outcome for symptomatic congenital CMV patients. (53, 54) However, $16 \mathrm{mg} / \mathrm{kg}$ of valganciclovir administered twice daily for 6 months did improve long term hearing and neurodevelopmental outcomes. (54) Furthermore, there have been two cases reporting effective utilization of Foscarnet in the management of congenital cytomegalovirus disease. (55) The use of foscarnet in congenital infections of CMV still lacks support to establish the efficacy of this drug in the pediatric population. (55) However, foscarnet is still considered to be the second line agent for pediatric neurological and herpes simplex virus infections. (55)

The treatment of congenital CMV patients presenting with isolated SNHL at birth has been a topic of controversy and general recommendations suggest against the use of antiviral therapy in such patients due to insufficient evidence. The results of a recent uncontrolled observational study have reported significant benefit of long-term antiviral therapy in patients with isolated SNHL. (56) The data from this study has also recorded no deterioration of hearing outcomes in the unaffected ear. (56) Interestingly, 8g/day of valaciclovir in pregnant women has also shown promising results in preventing transplacental CMV transmission after primary infection of the mother in the first trimester. (57) Implementation of this treatment strategy could limit symptomatic cases of congenital CMV infection.

\section{6 | CONCLUSION}

Congenital CMV infection is a clinically significant infection with a worldwide scope of prevalence. It is the principal non-genetic cause of sensorineural auditory impairment in children, and its symptomatically variable presentations pose an important challenge for clinicians since initial asymptomatic cases can also lead to debilitating sequelae months after birth. Various laboratory, ophthalmologic, and CNS findings can aid in the clinical identification of congenital CMV disease, and so, 
emphasis should be made on the accurate and timely diagnosis of this infection. Aptness in the diagnosis can help ascertain a well-timed initiation of the pharmacological and non-pharmacological interventions to aid the developmental outcomes of the child. Furthermore, ganciclovir, and valganciclovir can be administered after a risk-benefit evaluation of the patient. However, studies indicating the advantages of antiviral therapy, in the management of congenital CMV disease, have only highlighted therapeutic interventions concerning symptomatic cases, and there is still a lack of studies that focus on the therapeutic outcomes of these medications in asymptomatic patients and in patients with isolated SNHL. Therefore, a detailed study of the various aspects concerning asymptomatic congenital CMV infection is required. As of now, the use of antiviral medications is not indicated in silent CMV infections and treatment protocols may not even be investigated until indicators of debilitating clinical sequelae are established in these patients.

\section{7 | ACKNOWLEDGEMENTS}

We would like to express our gratitude to Muhammad Moin (Services Institute of Medical Sciences, Lahore), Muhammad Huzaifa (Services Institute of Medical Sciences, Lahore), and Iqra Nawaz (Quaid-e-Azam Medical College, Bahawalpur) for their technical support during the editing process. We would also like to extend our special thanks to our family and friends who have always motivated us to work on topics that we are passionate about.

\section{REFERENCES}

1. Sijmons S, Van Ranst M, Maes P. Genomic and functional characteristics of human cytomegalovirus revealed by next-generation sequencing. Viruses. 2014;6(3):1049-72. https://dx.doi.org/10.3390\%2Fv6031049

2. Syggelou A, lacovidou N, Kloudas S, Christoni Z, Papaevangelou V. Congenital cytomegalovirus infection. Ann N Y Acad Sci. 2010;1205:144-7. https://doi.org/10.1111/j.17496632.2010.05649.x

3. Dupont L, Reeves MB. Cytomegalovirus latency and reactiva- tion: recent insights into an age old problem. Rev Med Virol. 2016;26(2):75-89. https://doi.org/10.1002/rmv.1862

4. Hizel S, Parker S, Onde U. Seroprevalence of cytomegalovirus infection among children and females in Ankara, Turkey, 1995. Pediatr Int. 1999;41(5):506-9. https://doi.org/10.1046/j.1442200x.1999.01118.x

5. Forbes BA. Acquisition of cytomegalovirus infection: an update. Clin Microbiol Rev. 1989;2(2):204-16. https://dx.doi.org/10.1128\%2Fcmr.2.2.204

6. Roback JD. CMV and blood transfusions. Rev Med Virol. 2002;12(4):211-9. https://doi.org/10.1002/rmv.353

7. Pass RF, Anderson B. Mother-to-Child Transmission of Cytomegalovirus and Prevention of Congenital Infection. Journal of the Pediatric Infectious Diseases Society. 2014;3 Suppl 1(Suppl 1):S2-6. https://doi.org/10.1093/jpids/piu069

8. Pass RF, Zhang C, Evans A, Simpson T, Andrews W, Huang ML, et al. Vaccine prevention of maternal cytomegalovirus infection. $\mathrm{N}$ Engl J Med. 2009;360(12):1191-9. 10.1056/NEJMoa0804749.

9. Liesnard C, Donner C, Brancart F, Gosselin F, Delforge ML, Rodesch F. Prenatal diagnosis of congenital cytomegalovirus infection: prospective study of 237 pregnancies at risk. Obstet Gynecol. 2000;95(6 Pt 1):881-8. https://doi.org/10.1016/s00297844(99)00657-2

10. Boppana SB, Rivera LB, Fowler KB, Mach M, Britt WJ. Intrauterine transmission of cytomegalovirus to infants of women with preconceptional immunity. N Engl J Med. 2001;344(18):1366-71. https://doi.org/10.1056/nejm200105033441804

11. Gaytant MA, Rours GI, Steegers EA, Galama JM, Semmekrot BA. Congenital cytomegalovirus infection after recurrent infection: case reports and review of the literature. Eur J Pediatr. 2003;162(4):248-53. https://doi.org/10.1007/s00431-002-11155

12. Hamprecht K, Goelz R. Postnatal Cytomegalovirus Infection Through Human Milk in Preterm Infants: Transmission, Clinical Presentation, and Prevention. Clin Perinatol. 2017;44(1):121-30. https://doi.org/10.1016/j.clp.2016.11.012

13. Vochem M, Hamprecht K, Jahn G, Speer CP. Transmission of cytomegalovirus to preterm infants through breast milk. Pediatr Infect Dis J. 1998;17(1):53-8. https://doi.org/10.1097/00006454199801000-00012

14. Stagno S, Brasfield DM, Brown MB, Cassell GH, Pifer LL, Whitley RJ, et al. Infant pneumonitis associated with cytomegalovirus, Chlamydia, Pneumocystis, and Ureaplasma: a prospective study. Pediatrics. 1981;68(3):322-9.

15. Kadambari S, Whittaker E, Lyall H. Postnatally acquired cytomegalovirus infection in extremely premature infants: how best to manage? Archives of Disease in Childhood - Fetal and Neonatal Edition. 2020;105(3):334. https://doi.org/10.1136/archdischild2019-317650

16. Goldblum RM, Dill CW, Albrecht TB, Alford ES, Garza C, Goldman AS. Rapid high-temperature treatment of human milk. 
J Pediatr. 1984;104(3):380-5. https://doi.org/10.1016/s00223476(84)81099-9

17. Bryant P, Morley C, Garland S, Curtis N. Cytomegalovirus transmission from breast milk in premature babies: does it matter? Arch Dis Child Fetal Neonatal Ed. 2002;87(2):F75-7. https://doi.org/10.1136/fn.87.2.f75

18. Ronchi A, Zeray F, Lee LE, Owen KE, Shoup AG, Garcia F, et al. Evaluation of clinically asymptomatic high risk infants with congenital cytomegalovirus infection. J Perinatol. 2020;40(1):89-96. https://doi.org/10.1038/s41372-019-0501-z

19. Kashden J, Frison S, Fowler K, Pass RF, Boll TJ. Intellectual assessment of children with asymptomatic congenital cytomegalovirus infection. J Dev Behav Pediatr. 1998;19(4):254-9. https://doi.org/10.1097/00004703-199808000-00003

20. Fowler KB, Boppana SB. Congenital cytomegalovirus (CMV) infection and hearing deficit. J Clin Virol. 2006;35(2):226-31. https://doi.org/10.1016/j.jcv.2005.09.016

21. Zhang XW, Li F, Yu XW, Shi XW, Shi J, Zhang JP. Physical and intellectual development in children with asymptomatic congenital cytomegalovirus infection: a longitudinal cohort study in Qinba mountain area, China. J Clin Virol. 2007;40(3):180-5. https://doi.org/10.1016/j.jcv.2007.08.018

22. Kylat RI, Kelly EN, Ford-Jones EL. Clinical findings and adverse outcome in neonates with symptomatic congenital cytomegalovirus (SCCMV) infection. Eur J Pediatr. 2006;165(11):773-8. https://doi.org/10.1007/s00431-006-01726

23. La Torre R, Nigro G, Mazzocco M, Best AM, Adler SP. Placental enlargement in women with primary maternal cytomegalovirus infection is associated with fetal and neonatal disease. Clin Infect Dis. 2006;43(8):994-1000. https://doi.org/10.1086/507634

24. Boppana SB, Pass RF, Britt WJ, Stagno S, Alford CA. Symptomatic congenital cytomegalovirus infection: neonatal morbidity and mortality. Pediatr Infect Dis J. 1992;11(2):93-9. https://doi.org/10.1097/00006454-199202000-00007

25. Bale JF. Chapter 15 - Congenital cytomegalovirus infection. In: Tselis AC, Booss J, editors. Handb Clin Neurol. 123: Elsevier; 2014. p. 319-26.

26. Ouellette C, Ronchi A, Mejias A, Chavez-Bueno S, Salamon $\mathrm{D}$, Pugni L, et al. 998: Detection of Cytomegalovirus (CMV) in Cerebrospinal Fluid of Infants with Congenital CMV Infection: Is It Worth Doing the Lumbar Puncture?: Open Forum Infect Dis. 2014 Dec;1(Suppl 1):S292. doi: 10.1093/ofid/ofu052.706. Epub 2014 Dec.

27. Schleiss MR, Choo DI. Mechanisms of congenital cytomegalovirus-induced deafness. Drug Discov Today Dis Mech. 2006;3(1):105-13. https://doi.org/10.1016/j.ddmec.2006.02.009

28. Davis GL, Spector GJ, Strauss M, Middlekamp JN. Cytomegalovirus endolabyrinthitis. Arch Pathol Lab Med. 1977;101(3):118-21.

29. Fowler KB. Congenital cytomegalovirus infection: audiologic outcome. Clin Infect Dis. 2013;57 Suppl 4(Suppl 4):S182-4. https://doi.org/10.1093/cid/cit609

30. Lanzieri TM, Chung W, Flores M, Blum P, Caviness AC, Bialek SR, et al. Hearing Loss in Children With Asymptomatic Congenital Cytomegalovirus Infection. Pediatrics. 2017;139(3). https://doi.org/10.1542/peds.2016-2610

31. Foulon I, Naessens A, Foulon W, Casteels A, Gordts F. Hearing loss in children with congenital cytomegalovirus infection in relation to the maternal trimester in which the maternal primary infection occurred. Pediatrics. 2008;122(6):e1123-7. https://doi.org/10.1542/peds.2008-0770

32. Yoshinaga-Itano C. Early intervention after universal neonatal hearing screening: impact on outcomes. Mental retardation and developmental disabilities research reviews. 2003;9(4):252-66. https://doi.org/10.1002/mrdd.10088

33. Patel H, Feldman $M$. Universal newborn hearing screening. Paediatr Child Health. 2011;16(5):301-10. https://dx.doi.org/10.1093\%2Fpch\%2F16.5.301

34. Jin HD, Demmler-Harrison GJ, Coats DK, Paysse EA, Bhatt $\mathrm{A}$, Edmond JC, et al. Long-term Visual and Ocular Sequelae in Patients With Congenital Cytomegalovirus Infection. Pediatr Infect Dis J. 2017;36(9):877-82. https://doi.org/10.1097/inf.0000000000001599

35. Coats DK, Demmler GJ, Paysse EA, Du LT, Libby C. Ophthalmologic findings in children with congenital cytomegalovirus infection. J AAPOS. 2000;4(2):110-6. https://doi.org/10.1067/mpa.2000.103870

36. Noyola DE, Demmler GJ, Nelson CT, Griesser C, Williamson WD, Atkins JT, et al. Early predictors of neurodevelopmental outcome in symptomatic congenital cytomegalovirus infection. J Pediatr. 2001;138(3):325-31. https://doi.org/10.1067/mpd.2001.112061

37. Fink KR, Thapa MM, Ishak GE, Pruthi S. Neuroimaging of Pediatric Central Nervous System Cytomegalovirus Infection. 2010;30(7):1779-96. https://doi.org/10.1148/rg.307105043

38. Kawasaki H, Kosugi I, Meguro S, Iwashita T. Pathogenesis of developmental anomalies of the central nervous system induced by congenital cytomegalovirus infection. Pathol Int. 2017;67(2):7282. https://doi.org/10.1111/pin.12502

39. Ancora G, Lanari M, Lazzarotto T, Venturi V, Tridapalli $E$, Sandri $F$, et al. Cranial Ultrasound Scanning and Prediction of Outcome in Newborns with Congenital Cytomegalovirus Infection. The Journal of Pediatrics. 2007;150(2):157-61. https://doi.org/10.1016/j.jpeds.2006.11.032

40. Practice bulletin no. 151: Cytomegalovirus, parvovirus B19, varicella zoster, and toxoplasmosis in pregnancy. Obstet Gynecol. 2015;125(6):1510-25. https://doi.org/10.1097/01.aog.0000466430.19823.53

41. Beswick R, McHugh L, Clark JE. Integrating congenital cytomegalovirus screening within a newborn hearing screening program: Is it worthwhile? Int J Pediatr Otorhinolaryngol. 
2021;142:110594. https://doi.org/10.1016/j.ijporl.2020.110594

42. Williams EJ, Kadambari S, Berrington JE, Luck S, Atkinson C, Walter S, et al. Feasibility and acceptability of targeted screening for congenital CMV-related hearing loss. Arch Dis Child Fetal Neonatal Ed. 2014;99(3):F230-6. https://doi.org/10.1136/archdischild2013-305276

43. Courtmans I, Mancilla V, Ligny C, Le Bon SD, Naessens A, Foulon I. Incidence of congenital $\mathrm{CMV}$ in children at a hearing rehabilitation center. B-ent. 2015;11(4):303-8.

44. Cannon MJ, Griffiths PD, Aston V, Rawlinson WD. Universal newborn screening for congenital CMV infection: what is the evidence of potential benefit? Rev Med Virol. 2014;24(5):291-307. https://doi.org/10.1002/rmv.1790

45. Beswick R, David M, Higashi H, Thomas D, Nourse C, Koh G, et al. Integration of congenital cytomegalovirus screening within a newborn hearing screening programme. J Paediatr Child Health. 2019;55(11):1381-8. https://doi.org/10.1111/jpc.14428

46. Hughes BL, Gyamfi-Bannerman C. Diagnosis and antenatal management of congenital cytomegalovirus infection. Am J Obstet Gynecol. 2016;214(6):B5-b11. https://doi.org/10.1016/j.ajog.2016.02.042

47. Gantt S, Bitnun A, Renaud C, Kakkar F, Vaudry W. Diagnosis and management of infants with congenital cytomegalovirus infection. Paediatr Child Health. 2017;22(2):72-4. https://doi.org/10.1093/pch/pxx002

48. Lazzarotto T, Blázquez-Gamero D, Delforge ML, Foulon I, Luck S, Modrow S, et al. Congenital Cytomegalovirus Infection: A Narrative Review of the Issues in Screening and Management From a Panel of European Experts. Frontiers in pediatrics. 2020;8:13. https://doi.org/10.3389/fped.2020.00013

49. Bilavsky E, Watad S, Levy I, Linder N, Pardo J, Ben-Zvi H, et al. Positive IgM in Congenital CMV Infection. Clin Pediatr (Phila). 2017;56(4):371-5. https://doi.org/10.1177/0009922816684596

50. Guerra B, Lazzarotto T, Quarta S, Lanari M, Bovicelli L, Nicolosi A, et al. Prenatal diagnosis of symptomatic congenital cytomegalovirus infection. Am J Obstet Gynecol. 2000;183(2):47682. https://doi.org/10.1067/mob.2000.106347

51. Kimberlin DW, Lin CY, Sánchez PJ, Demmler GJ, Dankner $W$, Shelton $M$, et al. Effect of ganciclovir therapy on hearing in symptomatic congenital cytomegalovirus disease involving the central nervous system: a randomized, controlled trial. J Pediatr. 2003;143(1):16-25. https://doi.org/10.1016/s00223476(03)00192-6

52. Oliver SE, Cloud GA, Sánchez PJ, Demmler GJ, Dankner W, Shelton $\mathrm{M}$, et al. Neurodevelopmental outcomes following ganciclovir therapy in symptomatic congenital cytomegalovirus infections involving the central nervous system. J Clin Virol. 2009;46 Suppl 4(Suppl 4):S22-6. https://doi.org/10.1016/j.jcv.2009.08.012

53. Kimberlin DW, Acosta EP, Sánchez PJ, Sood S, Agrawal V, Homans J, et al. Pharmacokinetic and pharmacodynamic assessment of oral valganciclovir in the treatment of symptomatic con- genital cytomegalovirus disease. J Infect Dis. 2008;197(6):836-45. https://doi.org/10.1086/528376

54. Kimberlin DW, Jester PM, Sánchez PJ, Ahmed A, Arav-Boger R, Michaels MG, et al. Valganciclovir for symptomatic congenital cytomegalovirus disease. N Engl J Med. 2015;372(10):933-43. https://doi.org/10.1056/nejmoa1404599

55. Mareri A, Lasorella S, lapadre G, Maresca M, Tambucci R, Nigro G. Anti-viral therapy for congenital cytomegalovirus infection: pharmacokinetics, efficacy and side effects. J Matern Fetal Neonatal Med. 2016;29(10):1657-64. https://doi.org/10.3109/14767058.2015.1058774

56. Pasternak Y, Ziv L, Attias J, Amir J, Bilavsky E. Valganciclovir Is Beneficial in Children with Congenital Cytomegalovirus and Isolated Hearing Loss. The Journal of Pediatrics. 2018;199:166-70. https://doi.org/10.1016/j.jpeds.2018.02.028

57. Shahar-Nissan K, Pardo J, Peled O, Krause I, Bilavsky E, Bilavsky E, et al. LB20. Valacyclovir to Prevent Vertical Transmission of Cytomegalovirus After Maternal Primary Infection During Pregnancy. Open Forum Infect Dis. 2019;6(Suppl 2):S1002-S. https://dx.doi.org/10.1093\%2Fofid\%2Fofz415.2503 